\title{
Chemical Compositions of the Leaf Essential Oils of Aralia spinosa from Three Habitats in Northern Alabama
}

\section{Purva C. Davé, Bernhard Vogler, William N. Setzer}

Department of Chemistry, University of Alabama in Huntsville, Huntsville, USA.

Email: wsetzer@chemistry.uah.edu

Received May $7^{\text {th }}, 2011$; revised June $10^{\text {th }}, 2011$; accepted July $10^{\text {th }}, 2011$.

\begin{abstract}
Aralia spinosa leaves were collected from three different habitats in north Alabama. The leaf essential oils were collected by hydrodistillation and analyzed by gas chromatography/mass spectrometry (GC-MS). The most abundant components of A. spinosa essential oils were the sesquiterpenes germacrene D (28.0\% - 37.3\%), (E)-caryophyllene $(8.2 \%-15.7 \%)$, and $\alpha$-humulene $(1.9 \%-4.9 \%)$; the monoterpene myrcene (up to $15.1 \%)$, and the fatty-acid-derivative (2E)-hexenal (trace to 28.9\%). Fatty-acid derivatives and monoterpene hydrocarbons were more abundant in samples from suburban Huntsville than those from "natural" habitats (Monte Sano Mountain, Wheeler National Wildlife Refuge), while sesquiterpene hydrocarbons were more abundant in the natural/wild samples.
\end{abstract}

Keywords: Aralia Spinosa, Araliaceae, Leaf Essential Oil, Chemical Composition, Germacrene D, Myrcene, (E)-Caryophyllene, (2E)-Hexenal

\section{Introduction}

Aralia spinosa L. (Araliaceae), "devil's walking stick", is a large shrub/medium-sized (up to $10 \mathrm{~m}$ tall) tree native to the southeastern United States. The leaves are very large (up to $1.5 \mathrm{~m}$ long, $1 \mathrm{~m}$ wide), bipinnate with ovate leaflets, $2 \mathrm{~cm}-10 \mathrm{~cm}$ long. The plant produces numerous tiny white flowers in umbels (July-September) and bluish drupes [1]. To our knowledge, the leaf essential oil of $A$. spinosa has not been previously examined. In this work, we present and compare the chemical compositions of the leaf essential oils of $A$. spinosa from three habitats in north Alabama.

\section{Materials and Methods}

\subsection{Plant Material}

Leaves of A. spinosa were collected (July, 2010) from four mature trees growing in north Alabama: two trees from suburban Huntsville city $\left(34^{\circ} 38^{\prime \prime} 77^{\prime} \mathrm{N}, 86^{\circ} 33^{\prime \prime} 45^{\prime} \mathrm{W}\right.$, $187 \mathrm{~m}$ elevation), one tree from Monte Sano Mountain $\left(34^{\circ} 44^{\prime \prime} 85^{\prime} \mathrm{N}, 86^{\circ} 31^{\prime \prime} 77^{\prime} \mathrm{W}, 427 \mathrm{~m}\right.$ elevation), and one tree from Wheeler National Wildlife Refuge $\left(34^{\circ} 37^{\prime \prime} 40^{\prime} \mathrm{N}\right.$, $86^{\circ} 57^{\prime \prime} 08^{\prime} \mathrm{W}, 122 \mathrm{~m}$ elevation). The plants were identified and collected by P. Davé, and a voucher specimen has been deposited in the University of Alabama in Huntsville herbarium. The freshly chopped leaves from each plant were hydrodistilled using a Likens-Nickerson apparatus. Continuous extraction of the distillates with $\mathrm{CH}_{2} \mathrm{Cl}_{2}$ for three hours gave clear colorless essential oils (Huntsville \#1, 9.52\%; Huntsville \#2, 6.18\%; Monte Sano, 1.05\%; Wheeler NWR, 1.07\%).

\subsection{Gas Chromatography-Mass Spectrometry}

The leaf essential oils of $A$. spinosa were subjected to gas chromatographic-mass spectral analysis on an Agilent system consisting of a Model 6890 gas chromatograph, a Model 5973 mass selective detector [MSD, operated in the EI mode (electron energy $=70 \mathrm{eV}$ ), scan range $=45$ $\mathrm{amu}-400 \mathrm{amu}$, and scan rate $=3.99 \mathrm{scans} / \mathrm{sec}]$, and an Agilent ChemStation data system. The GC column was an HP-5ms fused silica capillary with a (5\% phenyl)-polymethylsiloxane stationary phase, film thickness of 0.25 $\mu \mathrm{m}$, a length of $30 \mathrm{~m}$, and an internal diameter of 0.25 $\mathrm{mm}$. The carrier gas was helium with a column head pressure of $48.7 \mathrm{kPa}$ and a flow rate of $1.0 \mathrm{~mL} / \mathrm{min}$. Inlet temperature was $200^{\circ} \mathrm{C}$ and interface temperature was $280^{\circ} \mathrm{C}$. The GC oven temperature program was used as follows: $40^{\circ} \mathrm{C}$ initial temperature, hold for 10 mins; in- 
creased at $3^{\circ} \mathrm{C} / \mathrm{min}$ to $200^{\circ} \mathrm{C}$; increased at $2^{\circ} \mathrm{C} / \min$ to $220^{\circ} \mathrm{C}$. A $1 \% \mathrm{w} / \mathrm{v}$ solution of the sample in $\mathrm{CH}_{2} \mathrm{Cl}_{2}$ was prepared and $1 \mu \mathrm{L}$ was injected using a splitless injection technique. Identification of the oil components was based on their retention indices determined by reference to a homologous series of $n$-alkanes, and by comparison of their mass spectral fragmentation patterns with those reported in the literature [2] and stored on the MS library [NIST database (G1036A, revision D.01.00)/ChemStation data system (G1701CA, version C.00.01.080)]. The percentages of each component are reported as raw percentages based on total ion current without standardization. The leaf essential oil compositions of $A$. spinosa are summarized in Table 1.

Table 1. Chemical compositions of Aralia spinosa leaf essential oils.

\begin{tabular}{|c|c|c|c|c|c|}
\hline \multirow{3}{*}{ RI } & \multirow{3}{*}{ Compound } & \multicolumn{4}{|c|}{ Percent Composition } \\
\hline & & \multicolumn{2}{|c|}{ Suburban Huntsville } & \multirow{2}{*}{$\begin{array}{c}\text { Monte Sano } \\
\text { Mountain }\end{array}$} & \multirow{2}{*}{$\begin{array}{c}\text { Wheeler } \\
\text { NWR }\end{array}$} \\
\hline & & $\# 1$ & $\# 2$ & & \\
\hline 760 & (2E)-Pentenal & 0.1 & 0.4 & 0.2 & --- \\
\hline 773 & (2Z)-Pentenol & 1.7 & 2.0 & 0.8 & --- \\
\hline 795 & (3Z)-Hexenal & $--^{\mathrm{a}}$ & 8.3 & --- & --- \\
\hline 800 & Hexanal & 2.8 & --- & 1.6 & $\operatorname{tr}^{\mathrm{b}}$ \\
\hline 833 & Furfural & --- & --- & 0.2 & --- \\
\hline 845 & Unidentified hexenal & --- & --- & 0.5 & --- \\
\hline 854 & (2E)-Hexenal & 24.7 & 28.9 & 13.8 & $\operatorname{tr}$ \\
\hline 911 & $(2 E, 4 E)$-Hexadienal & 0.2 & 0.6 & $\operatorname{tr}$ & --- \\
\hline 934 & $\alpha$-Thujene & --- & --- & $\operatorname{tr}$ & --- \\
\hline 935 & $\alpha$-Pinene & $\operatorname{tr}$ & 0.6 & 1.4 & --- \\
\hline 953 & Camphene & --- & --- & 0.1 & --- \\
\hline 978 & $\beta$-Pinene & --- & 0.1 & 0.3 & --- \\
\hline 992 & Myrcene & 13.9 & 15.1 & $\operatorname{tr}$ & --- \\
\hline 1004 & $\alpha$-Phellandrene & --- & --- & $\operatorname{tr}$ & --- \\
\hline 1010 & $\delta$-3-Carene & --- & --- & 0.3 & --- \\
\hline 1027 & Limonene & --- & 0.2 & --- & $\operatorname{tr}$ \\
\hline 1031 & Benzyl alcohol & 0.3 & $\operatorname{tr}$ & --- & $\operatorname{tr}$ \\
\hline 1043 & Phenylacetaldehyde & --- & --- & --- & $\operatorname{tr}$ \\
\hline 1087 & Terpinolene & $\operatorname{tr}$ & 0.2 & 1.1 & --- \\
\hline 1097 & Linalool & 0.5 & --- & 0.2 & $\operatorname{tr}$ \\
\hline 1184 & $p$-Cymen-8-ol & --- & --- & --- & $\operatorname{tr}$ \\
\hline 1189 & $\alpha$-Terpineol & --- & --- & --- & $\operatorname{tr}$ \\
\hline 1374 & $\alpha$-Copaene & $\operatorname{tr}$ & --- & $\operatorname{tr}$ & $\operatorname{tr}$ \\
\hline 1383 & $\beta$-Bourbonene & 0.1 & --- & 0.8 & 0.6 \\
\hline 1391 & $\beta$-Elemene & 0.7 & --- & 5.1 & 3.9 \\
\hline 1419 & (E)-Caryophyllene & 10.7 & 8.2 & 15.7 & 12.5 \\
\hline 1428 & $\beta$-Copaene & 0.2 & --- & 0.2 & 0.2 \\
\hline 1453 & $\alpha$-Humulene & 2.7 & 1.9 & 4.9 & 4.7 \\
\hline 1458 & (E)- $\beta$-Farnesene & --- & --- & 0.8 & 0.7 \\
\hline 1478 & $\gamma$-Muurolene & 0.2 & $\operatorname{tr}$ & 0.5 & 0.4 \\
\hline 1482 & Germacrene D & 37.3 & 28.6 & 30.2 & 28.0 \\
\hline 1487 & $(E)-\beta$-Ionone & 0.2 & $\operatorname{tr}$ & 0.3 & 0.4 \\
\hline 1494 & cis- $\beta$-Guaiene & --- & --- & 0.7 & 1.1 \\
\hline 1497 & Bicyclogermacrene & 0.9 & 0.4 & 1.4 & 1.4 \\
\hline 1501 & $\alpha$-Muurolene & --- & --- & 0.2 & 0.3 \\
\hline 1505 & Germacrene A & --- & --- & 2.0 & 3.6 \\
\hline 1510 & $(E, E)-\alpha$-Farnesene & 0.6 & 0.3 & 1.1 & 1.1 \\
\hline 1514 & $\gamma$-Cadinene & --- & --- & 0.6 & 0.9 \\
\hline
\end{tabular}




\begin{tabular}{|c|c|c|c|c|c|}
\hline \multirow{3}{*}{ RI } & \multirow{3}{*}{ Compound } & \multicolumn{4}{|c|}{ Percent Composition } \\
\hline & & \multicolumn{2}{|c|}{ Suburban Huntsville } & \multirow{2}{*}{$\begin{array}{c}\text { Monte Sano } \\
\text { Mountain }\end{array}$} & \multirow{2}{*}{$\begin{array}{c}\text { Wheele } \\
\text { NWR }\end{array}$} \\
\hline & & $\# 1$ & $\# 2$ & & \\
\hline 1516 & Cubebol & --- & --- & $\operatorname{tr}$ & 0.9 \\
\hline 1525 & $\delta$-Cadinene & 0.4 & 0.2 & 2.0 & 2.4 \\
\hline 1566 & (E)-Nerolidol & 1.9 & 1.2 & 5.9 & 10.4 \\
\hline 1575 & Germacrene D-4-ol & --- & --- & --- & 1.0 \\
\hline 1578 & Spathulenol & --- & --- & --- & $\operatorname{tr}$ \\
\hline 1581 & Caryophyllene oxide & --- & --- & 0.3 & 1.4 \\
\hline 1593 & Salvial-4(14)-en-1-one & --- & --- & --- & $\operatorname{tr}$ \\
\hline 1608 & Humulene epoxide II & --- & --- & --- & 0.2 \\
\hline 1614 & 1,10-di-epi-Cubenol & --- & --- & --- & 0.4 \\
\hline 1617 & Junenol & --- & --- & --- & 0.2 \\
\hline 1627 & 1-epi-Cubenol & --- & --- & --- & 0.4 \\
\hline 1635 & Caryophylla-4(12),8(13)-dien-5-ol & --- & --- & --- & $\operatorname{tr}$ \\
\hline 1641 & $\tau$-Muurolol & --- & --- & 2.0 & 5.2 \\
\hline 1645 & $\alpha$-Muurolol (= Torreyol) & --- & --- & 0.3 & 1.0 \\
\hline 1654 & $\alpha$-Cadinol & --- & --- & 2.5 & 5.5 \\
\hline 1659 & Unidentified oxygenated sesquiterpenoid & --- & --- & 0.2 & 0.7 \\
\hline 1664 & Unidentified oxygenated sesquiterpenoid & --- & --- & 0.4 & 1.2 \\
\hline 1669 & 14-Hydroxy-9-epi-(Z)-caryophyllene & --- & --- & --- & 0.8 \\
\hline 1679 & Unidentified oxygenated sesquiterpenoid & --- & --- & --- & 0.7 \\
\hline 1685 & Germacra-4(15),5,10(14)-trien-1 $\alpha$-ol & --- & --- & 0.4 & 1.8 \\
\hline 1689 & Shyobunol & --- & --- & 0.5 & 2.3 \\
\hline 1739 & Mint sulfide & --- & --- & --- & 0.1 \\
\hline 1760 & Cyclocolorenone & --- & 2.8 & --- & 0.4 \\
\hline 1798 & 14-Hydroxy- $\delta$-cadinene & --- & --- & --- & 0.1 \\
\hline 1953 & Palmitic acid & --- & --- & --- & 0.2 \\
\hline \multirow[t]{8}{*}{2900} & Nonacosane & --- & --- & --- & 1.1 \\
\hline & Total Identified & 99.8 & 100.0 & 98.6 & 95.6 \\
\hline & Fatty-acid-derivatives & 29.4 & 40.2 & 16.5 & 1.2 \\
\hline & Monoterpene hydrocarbons & 13.9 & 16.2 & 3.2 & 0.0 \\
\hline & Oxygenated monoterpenoids & 0.5 & 0.0 & 0.2 & 0.0 \\
\hline & Sesquiterpene hydrocarbons & 53.7 & 39.6 & 66.3 & 61.8 \\
\hline & Oxygenated sesquiterpenoids & 1.9 & 4.0 & 12.5 & 34.7 \\
\hline & Others & 0.5 & 0.0 & 1.0 & 0.5 \\
\hline
\end{tabular}

a“----" = not detected. ${ }^{\mathrm{b} \text { “tr" }}=\operatorname{trace}(<0.05 \%)$.

\section{Results and Discussion}

A total of 60 compounds were identified in the A. spinosa leaf oils, representing $95.6 \%-100 \%$ of the compositions. A. spinosa leaf oils from suburban Huntsville, Alabama, were composed largely of sesquiterpene hydrocarbons $(53.7 \%$ and $39.6 \%)$, dominated by germacrene $\mathrm{D}(37.3 \%$ and $28.6 \%),(E)$-caryophyllene $(10.7 \%$ and $8.2 \%)$, and $\alpha$-humulene (1.9\% and $2.7 \%$ ); fatty-acid derivatives (29.4\% and $40.2 \%)$, mostly ( $2 E)$-hexenal; and the monoterpene hydrocarbon myrcene (13.9\% and $15.1 \%)$. The samples growing wild in natural habitats had much reduced monoterpenes but increased concentrations of sesquiter- penoids. The most abundant leaf oil components in the Monte Sano sample were germacrene D (30.2\%), $(E)$-caryophyllene $(15.7 \%),(2 E)$-hexenal $(13.8 \%),(E)$-nerolidol (5.9\%), and $\alpha$-humulene (4.9\%). The Wheeler NWR sample of $A$. spinosa has similar concentrations of sesquiterpene hydrocarbons to the Monte Sano sample, but virtually no monoterpenoids or "green leaf" volatiles. It was composed, however, of a diverse array of oxygenated sesquiterpenoids including relatively large concentrations of $(E)$-nerolidol $(10.4 \%), \alpha$-cadinol $(5.5 \%), \tau$ muurolol (5.2\%), and shyobunol (2.3\%).

Essential oils from other members of the Araliaceae have been analyzed. Pinenes dominated the essential oils 
of Acanthopanax trifoliatus [3], Schefflera heptaphylla [4], and Aralia cachemirica [5], while $\delta$-3-carene was the most abundant component of Dendropanax capillaris [6]. The sesquiterpene hydrocarbons $(E)$-caryophyllene, $\alpha$ humulene, and germacrene D were the dominant components of Schefflera stellata [7], Schefflera rodrigueziana [6], and Oreopanax nubigenus [6]; (E)-nerolidol was abundant in both Pseudopanax discolor [8] and $O p$ lopanax horridus [9]; and (2E)-hexenal was a major component of Dendropanax gonatopodus [10]. The essential oils of Pseudopanax discolor and Pseudopanax lessonii had notable quantities of $\tau$-muurolol [8], while Dendropanax arboreus and Oreopanax xalapensis were rich in shyobunol [11].

\section{Acknowledgements}

PCD is thankful for the summer research stipend provided by the University of Alabama in Huntsville Research and Creative Experiences for Undergraduates (RCEU) program. WNS is grateful to an anonymous private donor for the generous gift of the GC-MS instrumentation.

\section{REFERENCES}

[1] C. L. Brown and L. K. Kirkman, "Trees of Georgia and Adjacent States," Timber Press, Portland, 1990, pp. 208-209.

[2] R. P. Adams, "Identification of Essential Oil Components by Gas Chromatography/Mass Spectrometry," 4th Edition, Allured Publishing, Carol Stream, 2007.

[3] A. Muselli, T. M. Hoi, L. D. Cu, L. D. Moi, J. M. Bessière, A. Bighelli and J. Casanova, "Composition of the Essential Oil of Acanthopanax trifoliatus (L.) Merr. (Araliaceae) from Vietnam," Flavour and Fragrance Journal, Vol. 14, No. 1, 1999, pp. 41-44. doi:10.1002/(SICI)1099-1026(199901/02)14:1<41:AID-F FJ781>3.0.CO;2-B
[4] Y. L. Li, C. M. Yeung, L. C. M. Chiu, Y. Z. Cen and V. E. C. Ooi, "Chemical Composition and Antiproliferative Activity of Essential Oil from the Leaves of a Medicinal Herb, Schefflera heptaphylla," Phytotherapy Research, Vol. 23, No. 1, 2009, pp. 140-142. doi:10.1002/ptr.2567

[5] R. S. Verma, R. C. Padalia, A. Yadav and A. Chauhan, "Essential Oil Composition of Aralia cachemirica from Uttarakhand, India," Records of Natural Products, Vol. 4, No. 3, 2010, pp. 163-166.

[6] J. D. Richmond, B. R. Agius, B. S. Wright, W. A. Haber, D. M. Moriarity and W. N. Setzer, "Essential Oil Compositions and Cytotoxic Activities of Dendropanax capillaris, Oreopanax nubigenus and Schefflera rodrigueziana from Monteverde, Costa Rica," Natural Product Communications, Vol. 4, No. 2, 2009, pp. 271-274.

[7] B. Sabulal, V. George, S. P. Nediyaparambu and M. Dan, "Volatile Oils from the Root, Stem and Leaves of Schefflera stellata (Gaertn.) Harms (Araliaceae): Chemical Characterization and Antimicrobial Activity," Journal of Essential Oil Research, Vol. 20, No. 1, 2008, pp. 79-82.

[8] R. J. Weston, "Essential Oils from the Leaves of Three New Zealand Species of Pseudopanax (Araliaceae)," Zeitschrift für Naturforschung, Vol. 59c, 2004, pp. 39-42.

[9] F. X. Garneau, G. Collin, H. Gagnon, F. I. Jean, H. Strobl and A. Pichette, "The Essential Oil Composition of Devil's Club, Oplopanax horridus J. E. Smith Miq.," Flavour and Fragrance Journal, Vol. 21, No. 5, 2006, pp. 792-794. doi:10.1002/ffj.1716

[10] W. N. Setzer, "Essential Oil Composition of Dendropanax gonatopodus from Monteverde, Costa Rica. An $a b$ initio Examination of Aromadendrane Sesquiterpenoids," Natural Product Communications, Vol. 3, No. 9, 2008, pp. 1557-1561.

[11] J. S. Werka, A. K. Boehme and W. N. Setzer, "Biological Activities of Essential Oils of Monteverde, Costa Rica," Natural Product Communications, Vol. 2, No. 12, 2007, pp. 1215-1219. 